\title{
Film DidATTICO E PEDAGOGIA DEL CINEMA IN ITALIA \\ NEL SECONDO DOPOGUERRA
}

\section{Federico Pierotti}

Riassunto: Il saggio presenta i principali temi che caratterizzano il dibattito sulla pedagogia del cinema in Italia tra la fine degli anni quaranta e l'inizio degli anni cinquanta. La prima parte ripercorre le diverse posizioni in relazione alla dimensione emotiva delle immagini cinematografiche, considerata da taluni come un ostacolo all'attività didattica, da altri come una base da sfruttare per una nuova forma di azione educativa. La seconda parte è dedicata al problema della comprensione delle strutture narrative e linguistiche del film in relazione al grado di sviluppo intellettivo dei giovani spettatori. Il dibattito nazionale evidenzia un terreno fortemente ricettivo nei confronti delle teorie metodologiche che negli stessi anni vanno maturando nell'ambito della filmologia. In questo senso, esso offre un'occasione di aggiornamento e di modernizzazione delle pratiche discorsive sul mezzo cinematografico.

A cavallo tra gli anni quaranta e cinquanta, il dibattito sulla pedagogia del cinema costituisce un campo discorsivo ampio e articolato all'interno del quale si confrontano personalità di diversa formazione e orientamento, dai professori universitari agli insegnanti, dai rappresentanti di istituzioni educative ai critici cinematografici. Le prospettive di osservazione risultano piuttosto eterogenee e assumono forme che spaziano dalla monografia al pamphlet, dal saggio all'articolo militante. Tentando una sintesi complessiva, è tuttavia possibile scorgere, nell'ampio corpus di testi di questo dibattito critico, il progressivo affermarsi sulla scena di un nuovo paradigma teorico, mutuato dalle acquisizioni della filmologia francese, interessata allo studio sperimentale degli effetti psicologici della proiezione cinematografica. Come rimarcava Alberto Marzi, "I cultori degli studi cinematografici hanno escogitato nuove teorie ed hanno creato una nuova scienza, cioè la filmologia, con una vastissima letteratura che va sviluppandosi specialmente in Francia, dove è nato tale studio, sulle ragioni profonde della suggestione che il cinema esercita sugli individui e sulle folle" ("Il cinema nell'educazione popolare" 244).

Affiancandosi e sostituendosi progressivamente all'ancora dominante paradigma ontologico, la nuova prospettiva metodologica impone di considerare in un'ottica scientifica, svincolata da preconcetti e moralismi di 
sorta, il problema della valenza psicologica, sociologica, culturale e infine anche didattica del mezzo cinematografico ${ }^{1}$. Nel contesto di una società caratterizzata dalla diffusione crescente dei media, il cinema, assieme alla radio e alla televisione, diviene un oggetto di indagine degno di un'attenzione particolare: "ormai ben strutturato nell'esperienza dell'uomo moderno, assume nella formazione psicologica individuale un ruolo di straordinaria importanza dalla cui conoscenza possono dipendere considerazioni pratiche di grande interesse" (Marzi, Canestrari 17). Un nuovo discorso specialistico tende ad affermarsi grazie alla presa di posizione di alcuni psicologi e pedagogisti interessati a includere il cinema all'interno del loro orizzonte di riflessione.

Diviene dunque sempre più avvertita la necessità di studiarne gli effetti su diversi possibili piani d'indagine psicologica: ad attirare una maggiore attenzione sono gli ambiti della percezione e dei fenomeni affettivi e, sebbene con un interesse più altalenante, quello della psicologia dell'intelligenza e della comprensione del film (R. Zazzo, "La comprensione del film" 146). Quali reazioni sono implicate nella situazione cinematografica? Quali sollecitazioni psicologiche essa può comportare? Come si differenzia l'esperienza dei bambini da quella degli adulti?

Assumendo a proprio punto di partenza le teorie che negli stessi anni vanno maturando nell'ambito della filmologia, gli studiosi che prendono parte al dibattito offrono risposte diversificate. Al di là delle differenze esistenti tra i diversi contributi, il dibattito sulla pedagogia del cinema mette tuttavia in evidenza un terreno particolarmente ricettivo nei confronti del nuovo paradigma metodologico che, proprio grazie alle riflessioni sugli effetti psicologici del cinema, va affermandosi sul piano internazionale, offrendo così un'occasione di aggiornamento e di modernizzazione delle pratiche discorsive sul mezzo cinematografico anche in Italia. In questo saggio intendo presentare alcuni dei temi che informano il dibattito nazionale sulla pedagogia del cinema tra la fine degli anni quaranta e l'inizio degli anni cinquanta, con particolare riferimento a due aspetti: la dimensione emotiva dell'immagine cinematografica e l'intelligibilità delle strutture narrative e linguistiche ${ }^{2}$.

1 Sui due paradigmi, ontologico e metodologico, che nel secondo dopoguerra vanno orientando le teorie del cinema, cfr. Casetti 15-22.

2 Sono invece esclusi dal presente saggio i pur numerosi contributi dedicati ai problemi sociologici o alle questioni legislative e istituzionali della cinematografia didattica. Per un quadro d'insieme sul cinema per ragazzi nel dopoguerra, cfr. Boero. 


\section{1. "Il grande passionario."}

\section{La dimensione emotiva dell'esperienza cinematografica}

Lo sforzo di considerare il potenziale educativo del cinema alla luce delle teorie maturate nei contesti disciplinari della pedagogia e della psicologia impone una nuova e diversa considerazione del film didattico. Coloro che negli stessi anni affrontavano il medesimo problema da una prospettiva ontologica tendevano piuttosto a interrogarsi sulla natura dei film didattici, sui modi di utilizzo, sul rapporto con la produzione, sulle strutture pubbliche o private che avrebbero dovuto supportarlo. Molti contributi di questo tipo ponevano l'attenzione su istanze definitorie e precisazioni terminologiche che sottendevano una concezione essenzialista del fenomeno: il film didattico tendeva a essere inquadrato con precisione e a essere distinto rigorosamente da altre categorie, come quelle del film spettacolare, del film scientifico, del film documentario. Il più significativo esponente di questa direttrice di indagine è senza dubbio Remo Branca, a lungo direttore della Cineteca scolastica e rappresentante per così dire istituzionale del problema della "cinedidattica" 3 . Branca era portato a considerare quest'ultima come un ambito autonomo e rigorosamente distinto da tutti gli altri, che in quanto tale presentava una serie di specificità (Questioni del cinema 161-189; La scuola e i film 37-65). Egli considerava il film didattico come un dispositivo educativo che poco aveva a che fare con il tipo di esperienza che gli alunni erano abituati a vivere nella sala cinematografica: nei suoi intendimenti infatti, i film da proiettare a scuola avrebbero dovuto essere muti, commentati direttamente dall'insegnante, e soprattutto completamente privi di elementi emotivi. A titolo di esempio, Branca citava la "cinelezione" intitolata Chi è Dio? (Vincenzo Sorelli 1945), da lui stesso ideata per scolari dai nove ai dodici anni ai fini dell'insegnamento del catechismo (La scuola e i film 103-126) 4 . Il cinema diventava in sostanza uno strumento didattico alternativo, una sorta di prolungamento della lanterna magica; esso poteva essere utile per stimolare l'interesse del bambino su contenuti storici e religiosi oppure su fenomeni scientifici e naturali adatti

3 Remo Branca, pittore e incisore, diresse a lungo la Cineteca scolastica, istituita nel 1938 come organo di Stato deputato alla promozione della cinematografia educativa. Fu direttore di diverse riviste di settore, come "Cinema d'oggi" (1949), "Il nuovo cinema" (1952, cambiò il titolo in "Lanterna" nel 1956) e autore di numerosi articoli e monografie dedicati alla cinedidattica (cfr. Boero 243-275).

4 Da non confondersi con l'omonimo cortometraggio catechistico realizzato sempre nel 1945 da Mario Soldati (cfr. Vanelli 1-54 e 105-110). 
all'illustrazione visiva. Il problema del film narrativo e delle sue peculiari dinamiche psicologiche, invece, non era oggetto di particolare interesse.

È proprio su questo punto che si può evidenziare la frattura più profonda tra le tesi essenzialiste di Branca e quelle degli psicologi e dei pedagogisti. La questione che stava più a cuore di questi ultimi era infatti proprio quella della partecipazione emotiva che il cinema era in grado di suscitare nei bambini. Benché le diverse prese di posizione al riguardo fossero articolate tra scetticismi ed entusiasmi, tutti gli specialisti concordavano sul fatto che l'indagine sul valore didattico del cinema dovesse partire da uno studio metodologicamente fondato e scientificamente validato sull'impatto psicologico del medium. In che misura le componenti emozionali del cinema avrebbero potuto contribuire a una qualche forma di progetto educativo? Le posizioni all'interno del dibattito si articolavano tra coloro che vedevano nel coinvolgimento emotivo un fattore non facilmente adattabile alle funzioni didattiche della scuola, e quelli che al contrario intravedevano nell'uso del cinema la possibilità di modernizzare i metodi d'insegnamento.

Tra i primi si possono collocare i pedagogisti Giovanni Calò e Luigi Volpicelli'6. A mo' di sintesi di questa posizione scettica si possono elencare alcune considerazioni di quest'ultimo, che invitava a riservare particolare attenzione al "carattere irrazionale e patico della comunicazione filmica" ("Cinema ed educazione" 10). Volpicelli proponeva una radicale opposizione tra cinema e azione educativa: "Se la parola, il discorso è l'elemento dell'educazione umana, onde la definizione famosa dell'umanesimo come il saper parlare agli uomini, e cioè, criterio, giudizio, libertà, insomma, e possesso di sé e del mondo, e perciò della verità, il cinematografo ne costituisce la più cospicua e decisiva antitesi, e per sua natura si afferma come il grande passionario" ("Pregiudiziali sul cinema e l'educazione" 57). Egli rimarcava che "Per un retto e reale uso del film didattico nella scuola, bisogna, dunque, non già adagiarsi sul valore visivo e intuitivo del film, ma

5 Giovanni Calò, professore di pedagogia all'Università di Firenze dal 1911 al 1952 e preside della facoltà di Lettere e filosofia dal 1944 al 1947, fu, tra l'altro, presidente del Centro didattico nazionale e dell'Associazione italiana di pedagogia (cfr. Ambrosoli su "Calò, Giovanni").

${ }^{6}$ Luigi Volpicelli diresse all'Università di Roma l'Istituto di pedagogia, dove a partire dal 1949 organizzò corsi di filmologia. Nel 1955 fondò la rivista I problemi della pedagogia. Collaborò al Centro di studi sociologici sul film (collegato al Centro sperimentale di cinematografia) insieme a Enrico Fulchignoni, Luigi Chiarini e Remo Branca. Collaborò anche alla sceneggiatura e ai dialoghi del film per ragazzi Orizzonti del sole (cfr. Boero 149-186). 
organizzare la cinematografia didattica, al pari di tutti gli altri strumenti didattici, per quanto essa, in molti casi, abbia un valore superiore a codesti altri strumenti" ("Cinema didattico e pedagogia” 38).

La principale preoccupazione è dunque quella di neutralizzare in qualche modo il portato di irrazionalità del cinema. A questo riguardo, Giovanni Calò sosteneva che le sollecitazioni emotive del film avrebbero attenuato le capacità critiche e indotto nel giovane spettatore uno stato di passività, sottoponendolo al rischio di soggiacere a comportamenti imitativi. Se nell'adulto tale fenomeno avrebbe potuto essere governato dalla piena padronanza delle facoltà intellettive, nel bambino il possesso ancora parziale di queste stesse facoltà avrebbe prodotto una pericolosa condizione di inerzia e di influenzabilità. Calò invitava a considerare le due facce del fenomeno: se da un lato la dimensione emotiva poteva essere utile a stimolare quella intellettuale, d'altra parte diventava necessario mantenere tale stimolo al di sotto di un certo grado di intensità, per evitare un'eccessiva enfatizzazione delle azioni rappresentate. La rapidità con cui esse si susseguono sullo schermo rallenterebbe le attività cognitive e intellettive del giovane spettatore, limitandone le capacità di riflessione e di giudizio, riducendone l'autocontrollo e la possibilità di staccarsi dal bombardamento degli stimoli7. Il pedagogista non escludeva la possibilità di un uso educativo del cinema, ma suggeriva che l'insegnante elaborasse strategie per controbilanciare la forza emotiva delle immagini in movimento, sollecitando negli alunni un'attitudine cognitiva nei confronti del film e trasformando così la proiezione in un'opportunità di riflessione. Il film diventava strumento di "educazione intellettuale" nel momento in cui il suo potere di fascinazione veniva sottomesso alle istanze della razionalità (Calò 156-165).

Sulla medesima lunghezza d'onda, sebbene con una posizione più spiccatamente propositiva, Raffaele Laporta ${ }^{8}$ suggeriva di aprire la didattica del cinema ai normali film spettacolari per creare occasioni di riflessione con gli alunni: a tale proposito, anche particolari generi di forte impatto emotivo, come il gangster movie o il film di guerra, sarebbero risultati utili per formare un'idea di giustizia capace di mettere in prospettiva gli impulsi violenti presenti nel mondo finzionale. Lidea di lezione sul film spettacolare

7 Sullo stato di debolezza indotto nei giovani dalla proiezione cinematografica, cfr. anche Gemelli.

8 Raffaele Laporta, pedagogista e insegnante di istituti secondari, fu direttore del Centro provinciale di cinematografia scolastica di Pescara. Fu inoltre responsabile dell'Istituto di pedagogia dell'Università La Sapienza dal 1970 al 1975. Nel 1957 pubblicò Cinema ed età evolutiva (cfr. Boero 187-210). 
avanzata da Laporta arrivava così a contemplare la proiezione di film solitamente considerati estranei alla didattica, come ad esempio I sabotatori (Saboteur, Alfred Hitchcock, 1942). Una tale necessità di "formare nell'individuo una mentalità critica nei confronti del film" 9 rappresentava un punto di convergenza per molti pedagogisti alla ricerca di una difesa nei confronti del potere suggestivo delle immagini cinematografiche. Questa convinzione trovava riscontro nelle teorie psicologiche che assimilavano la visione cinematografica allo stato ipnotico e onirico ${ }^{10}$. Enrico Fulchignoni, ad esempio ${ }^{11}$, rimarcava che:

la situazione di coscienza filmica presenta molti caratteri di affinità con la condizione ipnoide .... E in questa ultima condizione le reazioni emotive, liberate per così dire da qualsiasi controllo e favorite dallo stato crepuscolare dei poteri critici, tendono ad assumere una forza ed un livello espressivo che saranno di prezioso interesse per lo studio analitico dell'emotività. ("Filmologia e psicologia infantile" 32)

A tale riguardo, egli aveva notato sperimentalmente un rapporto diretto tra il ritmo delle immagini proiettate sullo schermo e le variazioni respiratorie (Fulchignoni 32-33).

In un altro dei suoi contributi, Fulchignoni assimilava l'esperienza cinematografica a quella onirica, in nome di una comune appartenenza al dominio del pensiero simbolico, tipico della sfera degli affetti, dei sentimenti e delle esperienze vissute. Al cinema, come nel sogno, le emozioni si distaccano dal loro oggetto principale e si associano a delle immagini, e tale associazione non avviene per corrispondenza logica, come nel pensiero razionale, ma "per raccostamento di emozioni simili, ognuna collegata ad un'immagine" ("Sul valore psicologico dell'immagine filmica" 40). E mentre nel sogno sono le emozioni a richiamare le immagini, nel cinema avverrebbe il contrario: "Nella situazione filmica, tale processo emotivo delle immagini simboliche si verifica sovra tutto con l'impiego del cosiddetto

9 Laporta, "Esempio di lezione sul film spettacolare", 125 (in corsivo nell'originale). 10 Per le teorie sul legame tra sogno e cinema, con particolare riferimento ad Agostino Gemelli, cfr. Bellavita, Locatelli 222-231.

11 Enrico Fulchignoni, laureato in medicina, alternò negli anni quaranta l'attività di regista teatrale e cinematografico a quella di insegnante al Centro sperimentale di cinematografia. Dal 1948 insegnò all'Istituto di psicologia dell'Università di Roma; in seguito, a Parigi, assunse la presidenza del Consiglio internazionale del cinema e della televisione dell'Unesco (cfr. Moneti su "Fulchignoni, Enrico"). 
materiale plastico, nella selezione, cioè di un materiale visivo e fotografabile il quale attui, nei suoi sviluppi e nella sua significazione il soggetto cinematografico" ("Sul valore" 40).

Anche secondo Cesare Musatti ${ }^{12}$, la rappresentazione cinematografica poteva essere collegata alla produzione di contenuti onirici, originando un particolare scambio sul piano subcosciente, in cui anche la fantasia assumeva valore di realtà. Questo scambio

da un lato si opera con la utilizzazione che l'inconscio processo formativo del sogno fa di materiali rappresentativi bell'e pronti, o facilmente modificabili, offerti da esperienze filmiche, e che dall'altro si attua per l'adesione - in occasione della osservazione di un film - di forti cariche emotive provenienti dall'inconscio alle situazioni filmiche così come sono da noi vissute (152-153).

In sostanza, per tutti quegli studiosi che tendevano a valutare negativamente questo forte investimento emotivo e subcosciente, il cinema si poneva come strumento educativo complesso e fortemente problematico. Il suo uso didattico sarebbe stato ammissibile entro determinati limiti, definiti dalla razionalizzazione. Altri psicologi e pedagogisti, come Alberto Marzi e Giuseppe Flores d'Arcais, erano invece più propensi a considerare la componente emotiva del cinema come uno strumento funzionale ai compiti di una pedagogia in grado di confrontarsi con i nuovi mezzi di comunicazione della modernità. Un maggiore coinvolgimento emotivo poteva allora diventare la base indispensabile per stimolare l'interesse necessario all'apprendimento e agevolare nel bambino nuovi modi di strutturare il mondo circostante. In questo senso, il cinema poteva offrire alle istituzioni educative possibilità non altrimenti realizzabili attraverso i mezzi d'insegnamento più tradizionali. Flores d'Arcais ${ }^{13}$, sostenitore della pedagogia attivistica, invitava a superare un'impostazione eccessivamente razionalistica, auspicando un metodo disposto a confrontarsi con il mondo emozionale e affettivo, nella cui piena realizzazione risiederebbe "la suprema istanza dell'opera educativa" (267). Solo stimolando l'interesse dell'alunno si sarebbe potuta realizzare una didattica attiva, non meramente pre-

12 Cesare Musatti, ordinario di psicologia all'Università di Milano, dedicò alcuni scritti ai rapporti tra cinema e psicanalisi (cfr. Casetti 174).

13 Giuseppe Flores d'Arcais (1908-2004), professore all'Università di Padova, fu uno dei pedagogisti più interessati alla riflessione sul cinema. Nel 1954 fondò e diresse la rivista Lumen, dedicata alla cinematografica scolastica, scientifica ed educativa (cfr. Boero 210-225). 
cettistica e dogmatica, più rispondente alle esigenze del mondo contemporaneo. Il pedagogista aggiungeva che "il recupero del mondo emotivo non può venire compiuto a discapito del mondo razionale" (Flores d'Arcais 267), ma d'altra parte sosteneva che un autentico dominio razionale sul mondo e su se stessi potesse essere ottenuto tenendo conto "della pienezza, della varietà della vita affettiva, passionale, di ogni singolo individuo" (Flores d'Arcais 270).

Per lo psicologo Alberto Marzi, il cinema doveva essere indagato in primo luogo nella prospettiva della psicologia della percezione ${ }^{14}$. Nelle sue argomentazioni, egli riprese molte delle tesi formulate dai filmologi francesi a questo riguardo. L'attrazione esercitata sui bambini dalle immagini in movimento dipendeva dal legame stretto e intimo da essi intrattenuto con la visibilità. Per questa stessa ragione "chiedere al cinema una funzione di insegnamento che si concluda nella conoscenza o nel dominio concettuale è contrario alle più elementari proprietà del suo linguaggio" (Marzi, Canestrari 24). Le strade indicate, tra gli altri, da Branca, da Calò e da Volpicelli venivano così messe in discussione, perché proprio attraverso l'evidenza percettiva delle immagini in movimento il cinema avrebbe avuto in sé la capacità di stimolare le esperienze sensoriali, rivelando capacità di penetrazione psicologica superiori a quelle dell'insegnante e aumentando al contempo le possibilità di memorizzazione. Lo psicologo faceva dipendere l'adesione intellettuale e affettiva dello spettatore dal fenomeno percettivo dell'impressione di realtà, introdotto in ambito filmologico dagli studi di Michotte e ripreso da altri studiosi italiani ${ }^{15}$. Lo studio delle condizioni dell'illusione di realtà portava a rimettere in discussione la scientificità dell'assimilazione agli stati ipnotici e onirici avallata da alcuni psicologi e psicanalisti.

Tale fenomeno, che per Michotte si produce immediatamente e incondizionatamente durante la proiezione cinematografica, non assopisce le capacità critiche, ma, al contrario, pone lo spettatore in un rapporto diretto con le immagini, realizzando uno stato di concentrazione sensoriale particolarmente intenso. Per i filmologi, lo statuto della realtà cinematografica si distingue da quello di altre realtà rappresentate (come quella teatrale o quella romanzesca): di fronte a una proiezione, infatti, da un lato lo spet-

14 Alberto Marzi, professore di psicologia presso le università di Bari e di Firenze, dedicò alcuni articoli allo studio degli effetti psicologici del cinema, della radio e della televisione.

15 Cfr. Marzi, "Presupposti psicologici"; Fulchignoni, "Sul valore psicologico dell'immagine filmica", "Filmologia e psicologia infantile"; Musatti. 
tatore ha l'impressione di percepire una realtà presente immediatamente vissuta, mentre dall'altro mantiene la consapevolezza circa il differente statuto psichico rispetto all'esperienza reale. Nel cinema, questi due stati coesistono in una forma conflittuale che viene risolta nel concetto di illusione. Lillusione consente infatti la dissociazione tra ciò che è percepito e ciò che è conosciuto: "La conseguenza psicologicamente più importante che ne deriva è che la realtà apparente (quella del movimento nel caso del cinema) non è per nulla affievolita dal fatto di 'sapere che si tratta d'una illusione" (Fulchignoni, "Filmologia e psicologia infantile" 30). Durante la proiezione, lo stimolo cinematografico attiva in primo luogo una reazione percettiva, mentre l'adesione affettiva (definita anche impressione di realtà psicologica) si produrrebbe solo successivamente, e sarebbe probabilmente favorita dalla prima.

René Zazzo affermava che davanti allo schermo "noi siamo ingannati percettivamente prima d'esserlo affettivamente" ("La comprensione del film" 148). Dunque, anche la capacità del cinema di indurre fenomeni di empatia, di identificazione e di imitazione si sarebbe dovuta considerare come una questione non direttamente connessa alle caratteristiche di base della situazione cinematografica, ma piuttosto a condizioni esterne preesistenti. In considerazione di questo, anche gli eventuali effetti suggestivi del film sul comportamento del giovane spettatore si sarebbero dovuti valutare alla luce di istanze già presenti nella sua personalità. L'importanza dei fattori soggettivi veniva particolarmente sottolineata da Evelina Tarroni ${ }^{16}$, in seguito ad alcuni esperimenti condotti all'Università di Roma: "sulla percezione del film, influiscono notevolmente i fattori dell'esperienza personale dei singoli spettatori, e ... sono in gran parte questi fattori a suscitare l'interesse dello spettatore allo spettacolo cinematografico" (Filmologia pedagogica 28). Il potere ipnotico che si sarebbe voluto attribuire alla proiezione non avrebbe avuto la capacità di indurre un forte interesse in chiunque, poiché il fenomeno si sarebbe in ogni caso fondato sulle possibilità di ancoraggio a condizioni patologiche già esistenti in un individuo o in un gruppo sociale: "Quando dunque si addita il cinema come una delle cause principali dell'abbassamento del livello morale della società moderna, si scambia in verità la causa per l'effetto perché, in realtà, quel dato cinema, come quella data letteratura non è che l'espressione, il sintomo di una data società" ("Sul cinema ricreativo per ragazzi" 52). Trovava così un netto ridi-

16 Evelina Tarroni collaborò all'Istituto di pedagogia dell'Università di Roma (diretto da Volpicelli) e con l'ufficio studi della Cineteca scolastica. Oltre a Filmologia pedagogica (1950), pubblicò anche, con Sandro Paderni, Cinema e gioventù (1952). 
mensionamento anche il problema delle influenze negative del cinema sul comportamento dei giovani e sulla delinquenza minorile, che tante preoccupazioni e levate di scudi continuava a suscitare nei settori più conservatori del mondo educativo ${ }^{17}$.

\section{Linguaggio cinematografico e comprensione del film}

Nel dibattito postbellico sulla pedagogia del cinema, la questione dell'intelligibilità del film fu trattata meno frequentemente di quella dell'affettività, per effetto della tendenza a considerare in prima istanza le ricadute morali dei fenomeni di spettatorialità infantile e adolescenziale (R. Zazzo, "La comprensione del film" 146). Seppure minoritarie, queste problematiche non furono del tutto trascurate, e, ancora sulla scorta della ricerca filmologica, furono trattate mediante gli strumenti offerti dalle coeve ricerche sulla psicologia dell'intelligenza. Uno dei temi maggiormente esaminati fu quello dell'evoluzione delle capacità di comprensione nelle diverse fasi dello sviluppo infantile: in quale misura i bambini erano in grado di capire le strutture spaziali e temporali elaborate dal linguaggio cinematografico? Alcuni esperimenti condotti attraverso la proiezione di filmati e la compilazione di questionari tendevano a confermare una certa difficoltà nel comprendere episodi il cui collegamento non fosse esplicitato in modo palese.

La filmologia aveva promosso questo tipo di indagine attraverso i lavori di Bianka e René Zazzo, di cui giunse eco anche nel dibattito nazionale. In una di queste esperienze erano stati mostrati a bambini con un diverso grado di sviluppo intellettivo tre diversi film scelti in base al grado di complessità in ordine alla rappresentazione dello spazio e del tempo, dei personaggi, degli oggetti e del concatenamento dell'azione ${ }^{18}$. Gli esiti dell'esperimento portarono a ipotizzare l'esistenza di un rapporto tra l'insorgere spontaneo dell'impressione di realtà, il grado di adesione affettiva e il livello di intelligibilità. Nei bambini tra i sei e i sette anni, l'impressione di

17 “Gli studiosi di criminologia infantile affermano che parecchi delitti sono stati commessi da ragazzi influenzati da films pericolosi, talvolta addirittura sotto la soggezione o psicosi derivata da visioni cinematografiche conturbanti ed immediatamente dopo la vista dello spettacolo, quasi sotto una suggestione ipnotica” (Rodighiero 16).

18 I risultati degli esperimenti di René e Bianka Zazzo furono pubblicati sulla Revue internationale de filmologie (cfr. R. Zazzo, "Niveau mental et compréhension du cinéma"; B. Zazzo, R. Zazzo, "Une expérience sur la compréhension du film”; B. Zazzo, “Analyse des difficultés d'une séquence cinématographique”). 
realtà sembrava favorire una prima forma di comprensione sincretica e globale dell'azione, legata alla percezione del movimento. La logica dell'azione risultava comprensibile soltanto se mostrata direttamente, mentre diventava man mano più complessa da decifrare nella misura in cui venivano a mancare l'omogeneità e la continuità dell'azione stessa. I bambini evidenziavano difficoltà crescenti man mano che questi due principi venivano trasgrediti: risultava ad esempio problematico riconoscere l'identità di un personaggio in seguito a un cambiamento di aspetto, stabilire l'identità di un luogo dopo un cambio del punto di vista, o ancora concatenare reciprocamente tempi e luoghi. Una piena capacità di inferire le connessioni di spazio, tempo e azione veniva invece registrata soltanto a partire dagli undici o dodici anni, quando, con l'acquisizione di forme più complesse di pensiero logico e relazionale, diventavano comprensibili le scelte sottese al taglio delle inquadrature, ai raccordi di montaggio e, più in generale, alla narrazione (R. Zazzo, "La comprensione del film" 148-149).

Un'indagine nella medesima direzione, spesso menzionata nel dibattito nazionale, fu compiuta, in collaborazione con l'Università di Ginevra, su quasi seicento ragazzi di età compresa tra gli otto e i quattordici anni. L'esperimento - di cui fu dato conto in un ampio articolo pubblicato su Bianco e nero - venne realizzato al fine di comprendere i meccanismi della visione cinematografica dei ragazzi. Il film prescelto fu il celebre documentario Nanouk l'esquimese (Nanook of the North, Robert J. Flaherty, 1922), realizzato quasi trent'anni prima. L'indagine rivelò le non poche difficoltà di comprensione dei bambini di fronte ad alcuni passaggi logici del film. Gli esiti dei questionari confermarono, soprattutto nei bambini più piccoli, una spiccata attitudine a una comprensione globale, mentre emergevano forti difficoltà nel riferire su singoli aspetti considerati isolatamente. Veniva così confermata l'idea che fosse necessario porre particolare attenzione nella scelta dei film da proiettare ai bambini, mostrando soltanto quelli caratterizzati da un'azione sviluppata in continuità (Albertini, Caruso).

Su quali dovessero essere questi film, le opinioni erano divergenti. Per alcuni, infatti, queste condizioni di intelligibilità erano garantite soltanto da una produzione specifica di film per i ragazzi, le cui strutture linguistiche, psicologiche e narrative fossero adeguate alla fascia di età. Evelina Tarroni, ad esempio, era di questo avviso: "Il film destinato ai bambini deve essere fatto ... su misura, cioè corrispondere ai reali interessi del mondo infantile, alle sue reali capacità di comprensione, alle sue reali possibilità emotive" ("Sul cinema ricreativo per ragazzi” 53). La visione di film non espressamente dedicati era sconsigliabile, poiché avrebbe sottoposto i bambini a uno sforzo eccessivo. Proprio nella prospettiva di facilitare la 
conoscenza e la diffusione di film per ragazzi, nel 1949 era nato in seno alla Mostra di Venezia il Festival internazionale del film per ragazzi; tra i moltissimi film rapidamente dimenticati, furono davvero pochi quelli di cui la storiografia avrebbe continuato a fare menzione, come il lungometraggio di animazione in Technicolor La rosa di Bagdad (Anton Gino Domeneghini) e Gli orizzonti del sole (Giovanni Paolucci), presentati e premiati rispettivamente nel 1949 e nel 195419.

Per i sostenitori della tesi opposta, sarebbe stato sufficiente selezionare i film più adatti tra quelli normalmente distribuiti nelle sale. Gli unici limiti avrebbero dovuto riguardare quella parte della produzione reputata inadatta per i contenuti trattati o per l'adozione di forme narrative troppo complesse. Marzi riteneva sufficiente che i film presentati ai bambini fossero adeguati alle loro capacità di comprensione ("Presupposti psicologici”). In questo senso, la familiarità con il cinema poteva diventare un elemento di per sé significativo, a monte di ogni considerazione o preoccupazione di carattere educativo. Per lo psicologo, infatti, il linguaggio cinematografico poteva essere considerato come una nuova forma di espressione logica, in grado di produrre modi peculiari di interpretazione della realtà. In questa prospettiva, il problema non era più solo quello di adeguare la scelta del film al grado di intelligenza dei bambini, ma di interrogarsi su come le strutture filmiche potessero interagire con lo sviluppo dell'intelligenza. Una intuitiva comprensione del linguaggio cinematografico, accentuata dall'assidua frequentazione di sale, avrebbe potuto influenzare il modo stesso di organizzare i dati del reale: "Si è constatato ... in ragazzi abituati ad assistere molto spesso a proiezioni cinematografiche e ad avere certe forme di istruzione audiovisiva, la tendenza ad impadronirsi e ad impiegare un nuovo metodo di strutturare la realtà secondo quello che viene suggerito dal linguaggio cinematografico" (Marzi, "Il cinema nell'educazione popolare" 245). Insomma, in fin dei conti il cinema non sarebbe stato utile solo come banco di prova per la psicologia dell'intelligenza, ma anche come importante strumento di autodidattica, capace di allargare la conoscenza e l'esperienza della realtà, di divenire esempio di relazioni umane e comportamenti sociali.

\section{Conclusioni}

La sintetica presentazione delle principali posizioni del dibattito sul valore pedagogico del cinema non pretende certo di esaurire un campo di indagine esteso e articolato, che richiederebbe ampliamenti e approfondimenti

19 Cfr. Boero 21-107. 
sia per quanto riguarda i temi trattati che i presupposti sottesi alle posizioni dei vari studiosi. Pur nella parzialità dello sguardo d'insieme qui tentato, è tuttavia possibile ricavare da quanto emerso alcune considerazioni riassuntive rispetto a questa arena teorico-critica, che - come si è visto coinvolge e appassiona molti studiosi e militanti, dando vita a una significativa fioritura di monografie, atti di convegno, riviste e altri contributi a carattere più occasionale.

L'indagine specialistica sul portato psicologico dell'esperienza cinematografica è un fenomeno inaugurato in Italia dagli scritti pionieristici di Mario Ponzo e Giuseppe D’Abundo, che negli anni dieci gettano le basi di una riflessione che godrà in patria di una fortuna assai limitata ${ }^{20}$. È soltanto con il dibattito del secondo dopoguerra sulla psicologia e sulla pedagogia nel cinema che molti dei problemi sollevati in precedenza sono considerati entro un quadro epistemologico più unitario, offerto dagli studi di ambito filmologico, e sono trattati nell'ottica di una più rigorosa sistematizzazione. Questo crescente interesse degli specialisti, dunque, può essere colto come il sintomo di un mutamento di scenario che impone un nuovo modo di studiare il cinema. Quest'ultimo, assieme ad altri media come la radio e la televisione, viene osservato in misura sempre maggiore attraverso gli strumenti metodologici offerti da ambiti disciplinari specifici: non solo la pedagogia e la psicologia, ma anche la sociologia, l'estetica, la linguistica. Pur nella diversa gradazione delle posizioni, la volontà di fondare il problema su prospettive scientifiche e sperimentali contribuisce al superamento delle posizioni basate sul senso comune e sul moralismo di impianto religioso. In questo senso, gli scritti qui indagati costituiscono un corpus sintomatico attraverso cui si possono cogliere due linee di tendenza tipiche di questi anni, e spesso strettamente congiunte: da un lato una progressiva laicizzazione del discorso sul valore pedagogico del medium, dall'altro una crescita di interesse da parte del mondo universitario nei confronti del cinema, che in una certa misura prelude alla sua successiva istituzionalizzazione come disciplina.

Questo dibattito evidenzia dunque l'appropriazione da parte degli studiosi di categorie concettuali e strumenti di indagine adeguati al contesto di una società in via di modernizzazione. Lasciandosi alle spalle i tecnicismi e le specificità di una concezione della "cinedidattica" come momento strumentalmente legato all'apprendimento di nozioni, gli studiosi più sintonizzati sui cambiamenti in atto nella società italiana impostano una riflessione di più ampio respiro sulla dimensione esperienziale dei media, 
non limitato al contesto dell'aula scolastica ma esteso all'orizzonte più ampio dei rapporti tra il film e lo spettatore. Un simile ampliamento contribuisce a introdurre nel dibattito nazionale, o quantomeno a ribadirne l'importanza, diverse questioni teoriche oggetto in questi anni della filmologia. Come ho accennato, si tratta di un primo tentativo di inserire in un quadro sistematico i problemi dell'emotività e dell'intelligibilità filmi$\mathrm{ca}$, che sarebbero rimasti al centro delle successive riflessioni teoriche sul cinema sia nell'ambito della semiologia e della psicanalisi che in quello del cognitivismo, fino ai più recenti sviluppi delle neuroscienze ${ }^{21}$.

Se da un lato il fenomeno qui indagato impone un aggiornamento di prospettive e di metodi d'indagine, d'altra parte va anche rilevato come esso non abbia avuto un seguito importante quanto le sue premesse avrebbero lasciato pensare. Dopo questo passaggio postbellico, infatti, la riflessione sulla pedagogia del cinema non ha offerto significativi contributi a nessuna di queste teorie, né d'altra parte sembra averne tratto particolari frutti. Nei decenni successivi, infatti, la pedagogia del cinema tenderà a diventare un campo poco praticato, oggetto quasi esclusivo di una produzione manualistica divisa tra approcci contenutistici e linguistici, ugualmente limitati sul piano delle aperture metodologiche e delle proposte teoriche.

\section{Università di Firenze}

\section{Opere citate}

Albertini, Laura, Caruso, Maria Pia. "Percezione e interpretazione di immagini cinematografiche nei ragazzi." Bianco e nero 10. 5 (1949): 9-27. Print.

21 Per la semiologia psicanalitica, che ha utilizzato le acquisizioni della filmologia per lo studio dei processi di identificazione cinematografica, si veda soprattutto il contributo di Metz. La ripresa dei modelli della psicologia cognitiva è invece alla base di uno dei filoni più significativi della teoria cinematografica dagli anni ottanta in avanti: in particolare, il problema della comprensione delle strutture narrative è al centro delle riflessioni cognitiviste sul cinema (si vedano ad esempio i contributi di Bordwell e Branigan). Per quanto riguarda lo studio delle emozioni, il primo lavoro che ha posto l'attenzione sulla possibilità di interpretare le immagini dal punto di vista delle reazioni emotive del pubblico è l'ormai classico contributo di Freedberg (Il potere delle immagini); lo stesso studioso ha poi ampliato il proprio orizzonte alla luce delle acquisizioni delle neuroscienze (Freedberg, "Empatia, movimento ed emozione", 13-67). Per un'introduzione al problema delle emozioni nella teoria del cinema, si vedano almeno Plantiga, Smith Editors, passim, "La natura delle emozioni", 7-16 (e il numero monografico della rivista Fata Morgana che lo contiene) e De Vincenti, Carocci, 9-21. 
Alovisio, Silvio, Mazzei, Luca, “'Materia grigia, sensibile ai segni...'. L'esperienza cinematografica nella riflessione psicologica italiana degli anni Dieci.” Figure della modernità nel cinema italiano (1900-1940). Eds. Raffaele De Berti, Massimo Locatelli. Pisa: ETS, 2008. 179-210. Print.

Ambrosoli, Luigi. "Calò, Giovanni”. Dizionario biografico degli italiani. Roma: Istituto della Enciclopedia Italiana, 1973. Vol. 16. 782-785. Print

Bellavita, Andrea, Locatelli, Massimo. "Padre Agostino Gemelli." Figure della modernità nel cinema italiano (1900-1940). Eds. Raffaele De Berti, Massimo Locatelli. Pisa: ETS, 2008. 211-234. Print.

Boero, Davide. All'ombra del proiettore. Il cinema per ragazzi nell'Italia del dopoguerra. Macerata: EUM, 2013. Print.

Bordwell, David. Narration in the fiction film. Madison: University of Wisconsin Press, 1985. Print.

Branca, Remo. Questioni del cinema. Roma: Biblioteca nuovo cinema, 1952. Print.

- La scuola e i film. Criterio e limiti della cinedidattica. Rovigo: Istituto padano di arti grafiche, 1952. Print.

Branigan, Edward. Narrative comprehension and film. London: Routledge, 1992. Print.

Calò, Giovanni. "Cinema ed educazione intellettuale." Atti del congresso internazionale sulla stampa periodica cinematografia e radio per ragazzi. 19-23 marzo 1952, Milano. Milano: Giuffrè, 1953. 156-165. Print.

Casetti, Francesco. Teorie del cinema, 1945-1990. Milano: Bompiani, 1993. Print.

De Vincenti, Giorgio, Carocci, Enrico. "Introduzione." Il cinema e le emozioni. Estetica, espressione, esperienza. Eds. Giorgio De Vincenti, Enrico Carocci. Roma: Ente dello Spettacolo, 2012. Print.

Flores d'Arcais, Giuseppe. Il cinema. Il film nella esperienza giovanile. Padova: Liviana, 1953. Print.

Freedberg, David. Il potere delle immagini. Il mondo delle figure: reazioni e emozioni del pubblico. Trad. di Giovanna Perini. Torino: Einaudi, 1993. Print.

"Empatia, movimento ed emozione." Immagini della mente: neuroscienze, arte, filosofia. Ed. Giovanni Lucignani. Milano: Raffaello Cortina, 2007. 1367. Print.

Fulchignoni, Enrico. "Sul valore psicologico dell'immagine filmica." Bianco e nero 9. 9 (1948): 28-43. Print.

—. "Filmologia e psicologia infantile." Bianco e nero 10. 12 (1949): 26-33. Print.

Gemelli, Agostino. "Gli adolescenti e il cinema”. Cinedidattica 3.1 (1952). 5-7. Print.

"La natura delle emozioni. Conversazione con David Freedberg" Ed. Alessia Cervini. Fata Morgana 4.12 (2010): 7-16. Print.

Laporta, Raffaele. "Esempio di lezione sul film spettacolare." Il film e i problemi dell'educazione. Ed. Luigi Volpicelli. Roma-Milano: Bocca, 1953. 121-131. Print.

. Cinema ed età evolutiva. Firenze: La nuova Italia, 1957. Print.

Marzi, Alberto. "Il cinema nell'educazione popolare". Atti del convegno nazionale su 'I problemi della formazione dei giovani lavoratori'. 16-18 settembre 1953, 
Bari. Bari: Ente Pugliese di Cultura Popolare e di Educazione Professionale, 1953. 17-27. Print.

"Presupposti psicologici che postulano l'uso dei mezzi audiovisivi per i fini dell'educazione”. I problemi della pedagogia 4.6 (1958): 920-931. Print.

Marzi, Alberto, Canestrari, Renzo. "La funzione psicologica del cinema nella educazione delle masse". Il film e i problemi dell'educazione. Ed. Luigi Volpicelli. Roma-Milano: Bocca, 1953. 17-27. Print.

Metz, Christian. Cinema e psicanalisi. Il significante immaginario. Trad. Daniela Orati. Venezia: Marsilio, 1980. Print.

Michotte Van der Berck, Albert. "Le caractère de 'réalité' des projections cinématographiques." Revue internationale de filmologie 1. 3-4 (1948): 249-261. Print.

Moneti, Guglielmo. "Fulchignoni, Enrico". Dizionario Biografico degli Italiani. Roma: Istituto della Enciclopedia Italiana, 1998. Vol. 50. 692-94

Musatti, Cesare. "I processi psichici attivati dal cinema". Atti del congresso internazionale sulla stampa periodica cinematografia e radio per ragazzi. 19-23 marzo 1952, Milano. Milano: Giuffrè, 1953. 150-156. Print.

Plantinga, Carl, Smith, Greg M. Editors. Passionate views: film, cognition, and emotion. Baltimore: The Johns Hopkins University Press, 1999. Print.

Rodighiero, Antonio. Il fanciullo di fronte al cinema. Vicenza: Libreria cattolica Al duomo, 1949. Print.

Tarroni, Evelina. "Sul cinema ricreativo per ragazzi." Bianco e nero 10. 10 (1949): 50-54. Print.

Filmologia Pedagogica. Milano: Viola, 1950. Print.

Tarroni, Evelina, Paderni, Sandro. Cinema e gioventù. Studio degli aspetti sociali e dei motivi d'interesse, Roma: Edizioni dell'Istituto, 1952. Print.

Vanelli, Marco. Chi è Dio? Storia del catechismo cinematografico di Mario Soldati, Diego Fabbri e Cesare Zavattini, Recco (GE): Le Mani, 2013. Print.

Volpicelli, Luigi. "Pregiudiziali sul cinema e l'educazione." Bianco e nero 10. 9 (1949): 51-60. Print.

—. "Cinema didattico e pedagogia." Bianco e nero, 10.12 (1949): 34-39. Print. . "Cinema ed educazione." Il film e i problemi dell'educazione. Ed. Luigi Volpicelli. Roma-Milano: Bocca, 1953. 9-13. Print.

Zazzo, Bianka. "Analyse des difficultés d'une séquence cinématographique par la conduite du récit chez l'enfant." Revue internationale de filmologie 3. 9 (1952): 25-36. Print.

Zazzo, Bianka, Zazzo, René. "Une expérience sur la compréhension du film." Revue internationale de filmologie 2. 6 (1951): 159-170. Print.

Zazzo, René. "Niveau mental et compréhension du cinéma." Revue internationale de filmologie 2. 5 (1951): 29-36. Print.

. "La comprensione del film in funzione del livello di evoluzione intellettuale del ragazzo". Atti del congresso internazionale sulla stampa periodica cinematografia e radio per ragazzi. 19-23 marzo 1952, Milano. Milano: Giuffrè, 1953. 146-149. Print. 


\section{FOTOGRAFIE}

Alcune riviste specializzate pubblicano nel corso degli anni cinquanta fotografie chiamate a documentare le reazioni emotive dei bambini al cinema. Sebbene nella maggior parte dei casi queste immagini siano pubblicate a scopi strumentali e propagandistici, esse offrono una testimonianza assai viva sulla disseminazione delle idee enunciate dagli psicologi e dai pedagogisti. Ne riproponiamo qui una selezione, accompagnata dalle didascalie con cui furono originariamente pubblicate.

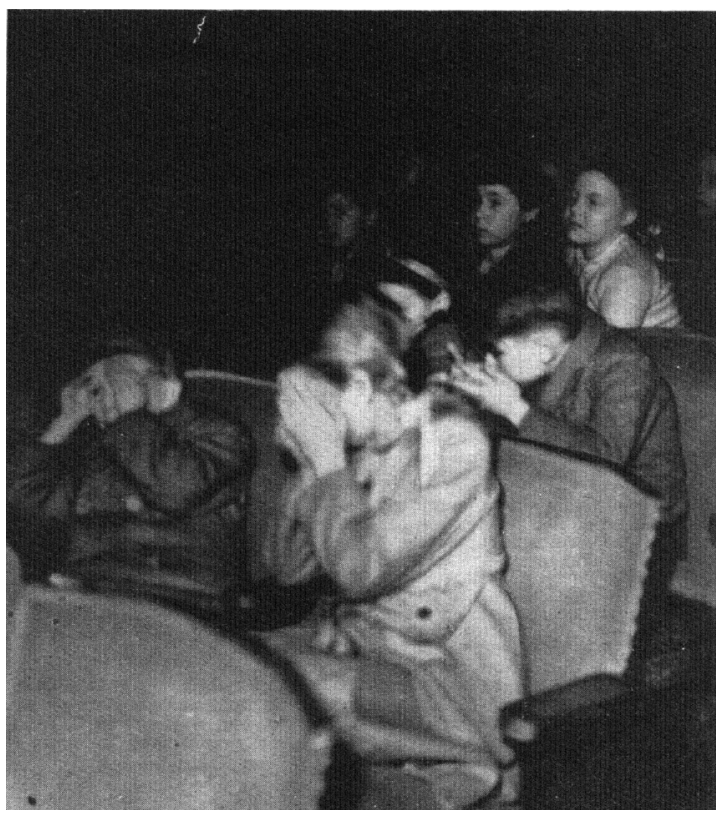

Foto 1. "Osservate il terrore che ha invaso l'anima di questi fanciulli di fronte a film che la censura giudica adatti per tutti" (Il nuovo cinema 2.9-10 [1953]: 30).



Foto 2. "Queste foto ai raggi infrarossi che hanno sorpreso la sofferenza dei bimbi al cinema per la proiezione di film non adatti alla loro età sono diventate celebri nel mondo, perché denunciano la devastazione che il cinematografo può portare e porta nella coscienza umana" (Il nuovo cinema 3.22 [1954]: 7). 


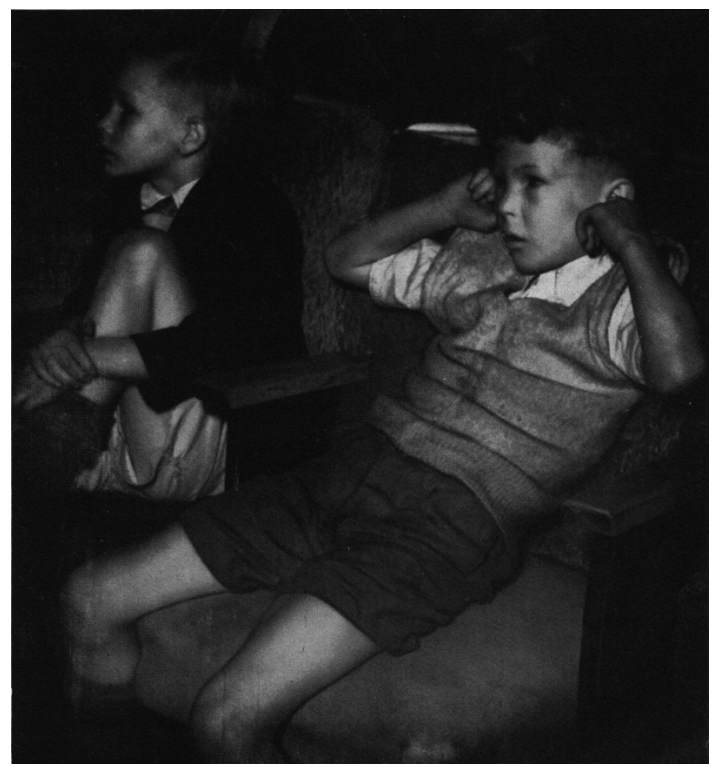

Foto 3. "Contro l'ingordigia del Capitale cinematografico che per ragioni puramente commerciali combatte il cinema dei ragazzi, le offese che il film 'per adulti' porta alla delicata anima dei ragazzi, sono una terribile realtà. La scuola italiana si è fatta promotrice di studi e di iniziative che ovunque si diffondono con successo crescente" (Il nuovo cinema 3.22[1954]: 11

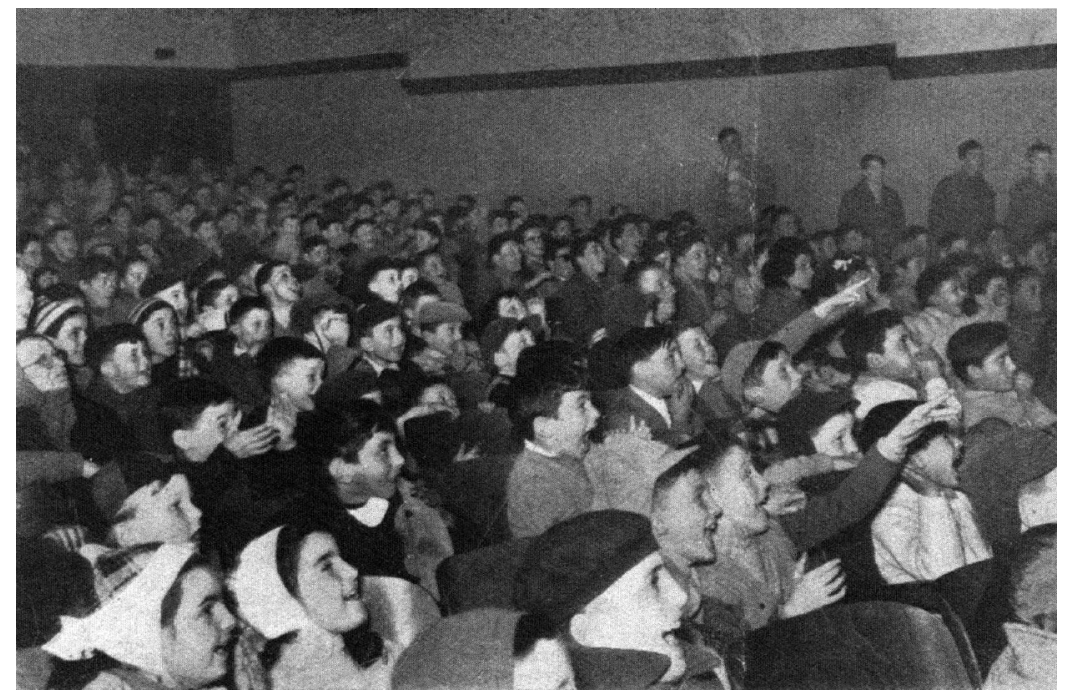

4. "A Cesena, durante una proiezione del 'Giovedì dei ragazzi' organizzato dal Centro Provinciale dei Sussidi Audiovisivi. I bambini manifestano vivacemente la loro partecipazione allo spettacolo" (Lanterna 6.6 [1957]: 7). 


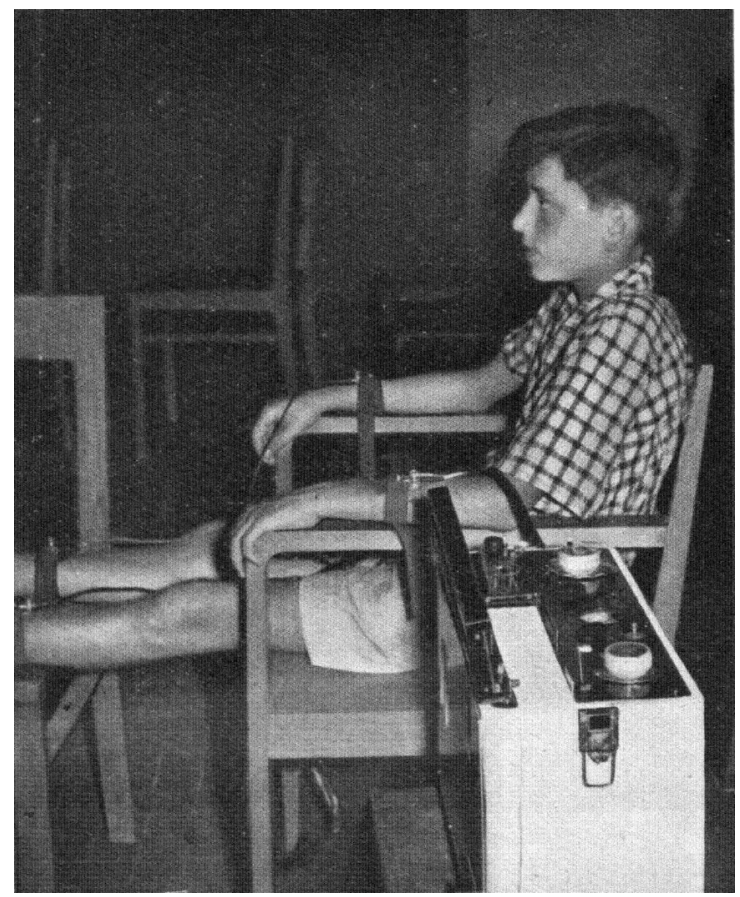

5. "Controllo delle reazioni del ragazzo durante la visione del film, attraverso l'elettrocardiogramma. Esperienza del prof. S. Gallo" (Lanterna 6. 910 [1957]: 12).

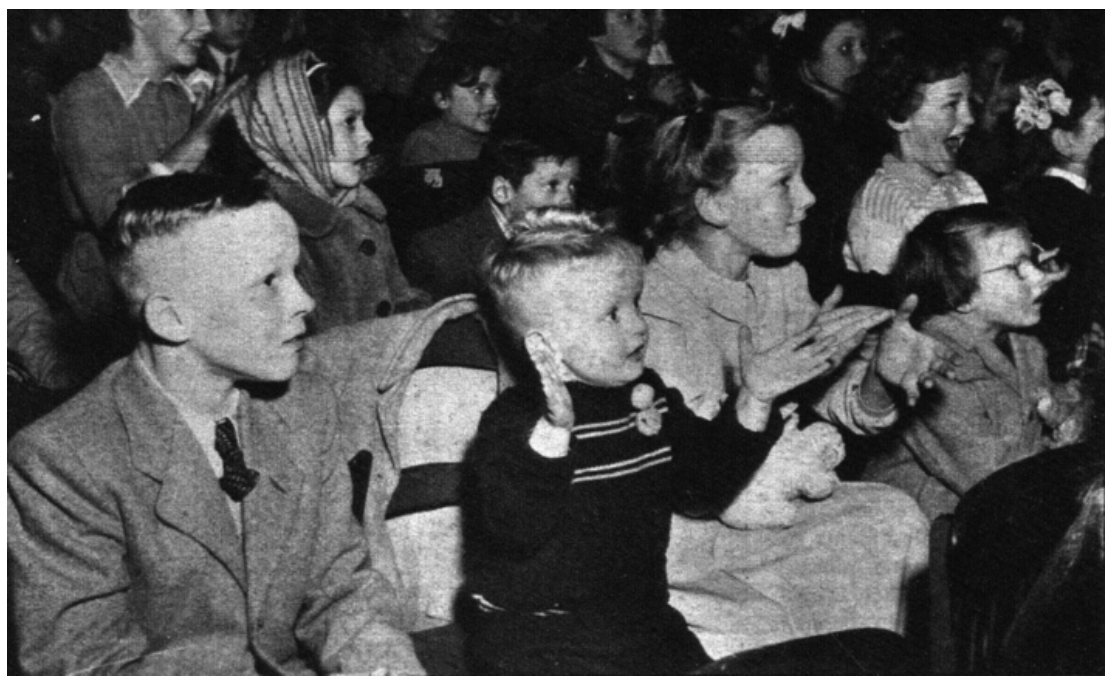

6. "Bambini al cinema: molteplici processi di identificazione che vanno dal semplice accompagnamento simpatetico con la vicenda fino all'incorporazione mimetica assoluta che spesso continua anche al di là della diretta esperienza filmica”. (Lanterna 7.8 [1959]: 10). 